\title{
El aprendizaje científico en el niño de preescolar: una experiencia dinámica
}

\section{Scientific knowledge in the preschool child: a dynamic experience}

\author{
BALDERAS-MIRELES, Ma. Dolores $\dagger^{*}$, ALMARAZ-OLGUÍN, Ma. Leticia, RAMÍREZ-VAQUERA, \\ Irma y BALDERAS-MIRELES, Kathia María Antonieta
}

Escuela Normal Experimental Rafael Ramírez Castañeda, México.

ID $1^{\mathrm{er}}$ Autor: Ma. Dolores, Balderas-Mireles / ORC ID: 0000-0002-7004-7184

ID $1^{\mathrm{er}}$ Coautor: Ma. Leticia, Almaraz-Olguín / ORC ID: 0000-0002-6574-2342

ID2 ${ }^{\text {do }}$ Coautor: Irma, Ramírez-Vaquera / ORC ID: 0000-0001-5924

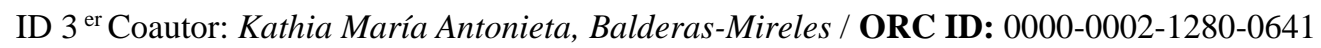

DOI: $10.35429 / J B E .2020 .12 .4 .19 .30$

Recibido 20 de Julio, 2020; Aceptado 30 de Diciembre, 2020

\begin{abstract}
Resumen
La investigación, se realizó a través de la sistematización y control de una experiencia de aula a partir de un enfoque cualitativo con la implementación de técnicas como observación, registro, videograbaciones y fotografías, cuyo resultado permitiera concientizar a los docentes de educación preescolar sobre la importancia del campo exploración y comprensión del mundo natural, la preponderancia de fomentar en el niño desde los tres años de edad habilidades como la observación, la curiosidad, la indagación, la experimentación y la formulación de hipótesis lo que demanda la preparación del maestro y un cambio de actitud hacia la enseñanza de las ciencias, así como el compromiso de las instituciones de nivel preescolar para fomentar el trabajo de este campo; el resultado de la propuesta de intervención impactó en habilidades más allá de las consideradas, pues el niño avanza conforme se le generan las condiciones para hacerlo y su pensamiento se expande hasta lograr argumentar a partir de lo que sabe y observa en relación con las experiencias vivenciales que el docente genera para ello.
\end{abstract}

Concimiento científico, niño, experimentación

\begin{abstract}
The research was carried out through the systematization and control of a classroom experience from a qualitative approach with the implementation of techniques such as observation, recording, video recordings and photographs, the result of which would make preschool teachers aware of the importance from the field of exploration and understanding of the natural world, the preponderance of fostering in the child from three years of age skills such as observation, curiosity, inquiry, experimentation and the formulation of hypotheses, which requires the preparation of the teacher and a change attitude towards science teaching, as well as the commitment of preschool institutions to promote work in this field; The result of the intervention proposal impacted on skills beyond those considered, as the child advances as the conditions to do so are generated and his thought expands until he can argue based on what he knows and observes in relation to experiential experiences that the teacher generates for it.
\end{abstract}

Scientific knowledge, Child, Experimentation

Citación: BALDERAS-MIRELES, Ma. Dolores, ALMARAZ-OLGUíN, Ma. Leticia, RAMÍREZ-VAQUERA, Irma y BALDERAS-MIRELES, Kathia María Antonieta. El aprendizaje científico en el niño de preescolar: una experiencia dinámica. Revista de Educación Básica. 2020. 4-12:19-30.

$\lceil$ Investigador contribuyendo como primer autor. 


\section{Introducción}

A los niños pequeños les interesa todo, les gusta observar y descubrir por sí mismos, aunque ello implique poner en riesgo su seguridad, cosa que los niños a temprana edad no logran comprender a la perfección, les gusta preguntar no por molestar sino porque quieren ampliar sus conocimientos y saber cosas y conceptos nuevos. El desarrollo del conocimiento científico infantil es un tema importante y una competencia que no debe postergarse para años superiores de escolarización, sino que, desde que son pequeños deben potenciarse este conjunto de habilidades, actitudes y destrezas que permita al futuro ciudadano participar de manera más consiente en su mundo desde una visión crítica, profunda y estudiada de las situaciones, dicha competencia sienta sus bases en la educación infantil, por ello la trascendencia e impacto de la investigación.

Trabajar las ciencias a través de distintas situaciones de aprendizaje como experimentos, observaciones, salidas de campo y ferias de la ciencia implicó la preparación del docente sobre los contenidos a abordar, así como la forma de trabajarlos a partir del análisis de los aprendizajes esperados que se seleccionaron. Promover el trabajo del campo Exploración y Comprensión del Mundo Natural es necesario y tan importante como matemáticas o lenguaje, desafortunadamente se ha descuidado y el poco trabajo que se hace por parte de los docentes de este nivel educativo no se hace a conciencia y con la metodología pertinente.

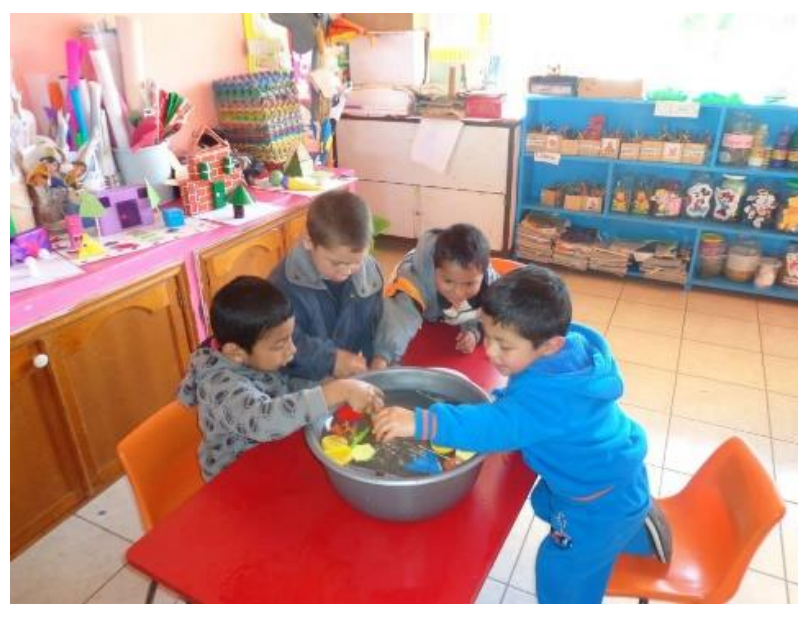

Figura 1 Las experiencias de aprendizaje para los niños deben permitir que manipulen los objetos de estudio
Con la propuesta de intervención se pretendió potenciar, en los niños de preescolar a través de la estrategia POE (predecir, observar y explicar) las habilidades como observación, curiosidad, indagación y exploración entre otras, ayudando con ello a la construcción de la visión empírica de los conocimientos de los alumnos.

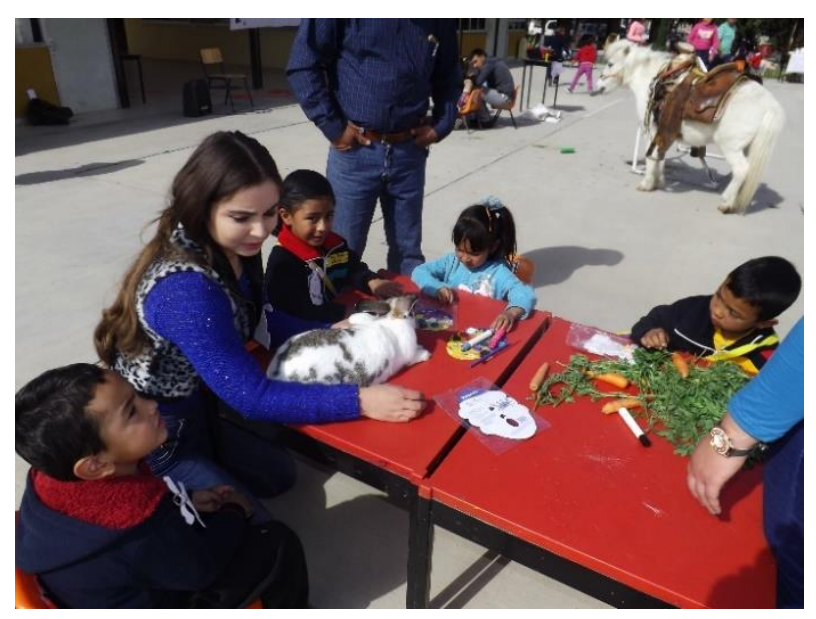

Figura 2 Las experiencias de los niños contribuyen al desarrollo del pensamiento científico

Con dicha experiencia se comprueba que los ambientes y oportunidades que se generan dentro y fuera del jardín de niños con la intención de promover el conocimiento científico infantil, impacta positivamente en el desarrollo de habilidades científicas en los niños, de igual manera se prueba la hipótesis: Entre mayor sea el apoyo dentro y fuera de la institución en el proceso de aprendizaje de los niños, mayor será el logro de los estándares curriculares y el desarrollo de la concepción científica que desarrollen.

En las diferentes secciones se teoriza y se expone la experiencia sobre desarrollo del conocimiento científico en el aula con niños de tres años, en la metodología expone el proceso que se siguió para sistematizar y recoger la información, en la última sección se da cuenta finalmente de los resultados que se obtienen, las conclusiones muestran las reflexiones a las que se llega con el estudio respecto al desarrollo de las habilidades científicas en los niños que en conjunto conforman el conocimiento científico, para cerrar el artículo se dejan los referentes bibliográficos utilizados para sustentar la experiencia. 


\section{El aprendizaje científico en los niños pequeños}

El aprendizaje científico va más allá del conocimiento de las matemáticas o de la naturaleza o en sí de la propia ciencia, es la implicación encaminada a la resolución de problemas que se le presentan al alumno de manera cotidiana, no pensemos cuando hablamos de aprendizaje científico sólo en las personas que se desenvuelven en un laboratorio entre batas blancas e instrumental científico, utilizando reactivos químicos y descubriendo en el día a día algo nuevo que revoluciona su quehacer cotidiano, que realizan sólo pruebas de tipo experimental científico.

Ampliemos nuestra visión y pensemos en las amas de casa que realizan ciencia en sus cocinas mezclando y transformando ingredientes, pensemos también en los artesanos que de manera cotidiana experimentan con una gran variedad de materiales en la realización de sus creaciones artísticas, pensemos en los herreros al transformar el hierro al tener contacto con el fuego y moldearlo con sus herramientas, transportémonos al trabajo que realizan los jardineros y agricultores, en los trabajadores del campo, pensemos en un sinfín de profesionistas $\mathrm{y}$ de obreros que realizan su trabajo ingeniosamente utilizando la ciencia.

Pensemos en los niños y niñas que de manera frecuente realizan actividades científicas sin pensar que es "ciencia" lo que realizan, para ellos simplemente es una actividad lúdica que más tarde se puede convertir en un proceso de creación e invención que puede revolucionar el mundo que los rodea en el cual están inmersos.

En este proceso los niños ponen en juego todas sus capacidades de retención, automotivación y sobre todo la autonomía para la búsqueda de sus propias estrategias al ponerlas en práctica, al igual buscan las soluciones a lo que se les presenta de manera cotidiana. El razonamiento y la lógica son dos capacidades que en la infancia se desarrollan como parte de un simple juego, pero a la vez son parte del aprendizaje científico, son herramientas que servirán para la resolución de problemas, para la adaptación ante variadas situaciones y para el enfrentamiento de los retos de la vida que se presentarán de manera cotidiana.
El propósito de enseñar ciencias en preescolar es desarrollar la capacidad del niño para entender la naturaleza de su entorno. Los niños y los adultos deben comprender que lo que se necesita para ser científico se tiene potencialmente en cada uno, independientemente del área en la que nos desarrollamos y nos desenvolvemos de manera cotidiana, "la capacidad que tienen los niños de aprender ciencias se basa en el conocimiento causal que tienen del mundo natural y en la posibilidad de diferenciar entre fuentes de conocimiento" (Quintanilla, G. M., 2017).

El objetivo de enseñar ciencias no es formar gente que vaya a dedicar el resto de su vida a cuestiones científicas, a estar sentado usando un microscopio o unos tubos de ensaye o reactivos manipulables con guantes por su alto grado de toxicidad, la intención principal es formar seres humanos con una visión integral, promoviendo el desarrollo de habilidades de pensamiento científicos en los niños; como el cuestionar y reflexionar sobre los fenómenos naturales que suceden a nuestro alrededor, mediante la investigación de las causas que los generan; esto permitirá al niño desarrollar una actitud diferente ante la ciencia y convertirse en un ser reflexivo, crítico y analítico; capaz de seguir un método para realizar investigaciones a partir de la información disponible, formular hipótesis y verificar las mismas mediante la experiencia. (Manual de experimentos, 2007)

Los niños de preescolar tienen nociones de lo que es el aprendizaje científico, ya que pueden hacer predicciones de lo que sucede y del por qué, al igual son capaces de formular preguntas y de buscar explicaciones del mundo que les rodea.

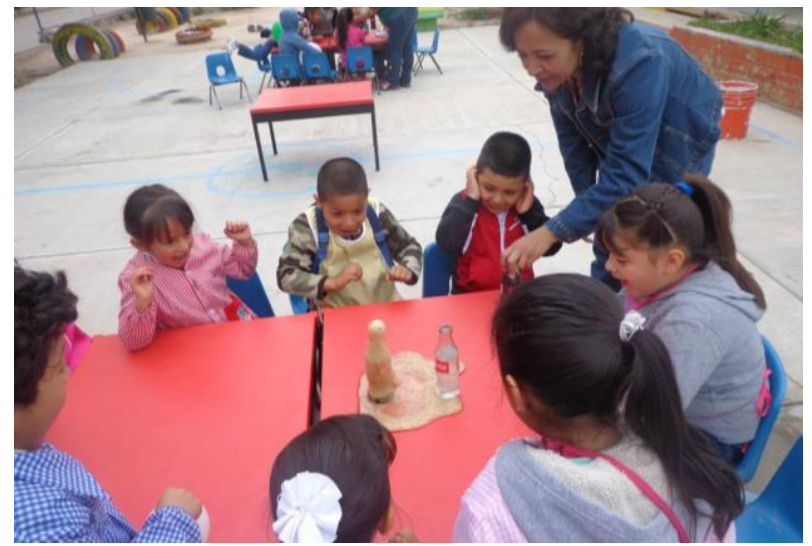

Figura $3 \mathrm{La}$ capacidad de asombro de los niños se observa en las actividades experimentales

BALDERAS-MIRELES, Ma. Dolores, ALMARAZ-OLGUÍN, Ma. Leticia, RAMÍREZ-VAQUERA, Irma y BALDERAS-MIRELES, Kathia María Antonieta. El aprendizaje científico en el niño de preescolar: una experiencia dinámica. Revista de Educación Básica. 2020 
La curiosidad espontánea y sin límites y la capacidad de asombro que los caracteriza, los lleva a preguntar constantemente cómo y por qué ocurren los fenómenos naturales y otros acontecimientos que llaman su atención, y a observar y explorar cuanto pueden usando los medios que tienen a su alcance. (SEP, Programa de Educacion Preescolar 2011 Guía para la educadora, 2011)

El niño desde que nace llega con la intención de explorar, algunos investigadores se atreven a decir que somos científicos desde la cuna, ese interés por explorar se convierte con el tiempo, en deseo incansable por curiosear. Lo que en un principio se satisface a través del sentido de la vista posteriormente se externa a través del aula. Varios pedagogos han estudiado el tránsito de ese proceso, Piaget citado por Meece (2001) en su clasificación a partir de los estadios señala que en el preoperatorio comprendido entre los dos y los siete años el niño fortalece el pensamiento intuitivo.

Ausbel, D. (1983) hace referencia al desarrollo del aprendizaje científico en niños pequeños a través de la construcción de conceptos por descubrimiento.

\section{Ideas previas de los niños}

Pero, cómo logra el niño el acercamiento al aprendizaje científico intencional, una vez que ha llegado al prescolar, Tonucci, F. (1995) se encontró que los conocimientos previos que tienen los niños en edad preescolar en relación con el aprendizaje científico son de crucial relevancia para el maestro ya que en base a ello se organiza el trabajo que se va a realizar, es de vital importancia tomar como base las ideas y concepciones que los niños y niñas tienen en relación a cómo ven el mundo que les rodea, a cómo explican los fenómenos naturales, a las respuestas e interrogantes que hacen a otros y para sí mismo.

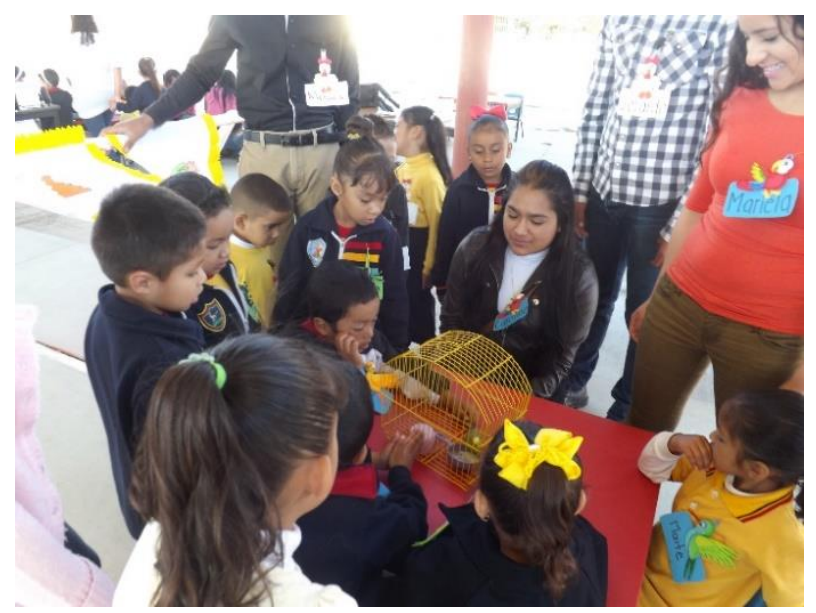

Figura 4 Las ideas previas de los niños se van modificando a partir de las oportunidades de aprendizaje que le brinde el maestro

Desde el punto de vista de Martín del Pozo R. (2013) las ideas de los alumnos tienen tres posibles orígenes: un origen sensorial que da lugar a concepciones de naturaleza espontánea; un origen social que da lugar a concepciones socialmente inducidas sobre todo a través de los medios de comunicación; un origen escolar que da lugar a concepciones inducidas por la enseñanza.

Otro de los pedagogos que hace su aportación es Vygotsky (1987) quien explica en su concepto de la "zona de desarrollo próximo" cómo no se avanza solamente partiendo de lo que el niño ya sabe, sino a partir de interacciones nuevas con personas adultas o más expertas. En esta medida la familia y el trabajo que se realiza dentro de ella en este aspecto incide en las ideas que los niños tienen acerca de tal o cual fenómeno o concepción del mundo y lo externan usando sus propias palabras, procesando lo que sus padres y otros miembros de la familia aportan a su conocimiento científico.

Desde la concepción anterior las ideas de los niños se ven influenciadas por el mundo que los rodea, al respecto Martín del Pozo (2013) considera como factores que influyen en las concepciones científicas de los alumnos: el medio, es decir, la propia experiencia de los alumnos sobre los fenómenos físico-naturales. 
Otro es la interacción social, es decir, los alumnos adoptan modos de pensar y de expresarse propios de una cultura y que interaccionan con sus experiencias, por último, considera también la transmisión cultural, es decir, la intervención del profesor como mediador entre sus propias experiencias, la interacción social y la ciencia como conocimiento público construido socialmente.

Sin duda se reviste la importancia que tienen que el niño se vuelva social, es innegable que el aprendizaje se acreciente; al respecto se reconoce la responsabilidad del profesor de crear lazos para la participación de la familia, es primordial que posibilite una interacción recíproca que propicie un trabajo en equipo y que contribuya al mejoramiento del aprendizaje del alumno/a.

No se desconoce el valor de las ideas previas que tienen los niños como un recurso didáctico valioso para propiciar aprendizajes nuevos dentro y fuera del salón de clases.

La doctora Bello, S. (2004) habla acerca de las ideas previas de los niños en relación con el aprendizaje científico, el cambio conceptual producto no sólo de la comprobación o sustitución de sus ideas es en parte producto de la interacción con el medio social en el cual se desarrolla el niño. En el modelo investigativo se priorizan estas ideas previas, se basa en la teoría constructivista, en la cual, la actividad del alumno es esencial para "... la búsqueda de explicaciones más o menos formalizadas de las prácticas docentes" (Porlan, 1989).

Tanto para la ciencia como para la escuela las ideas previas de los niños son o pueden ser errores, pero desde el punto de vista del niño y considerando su experiencia, estas ideas son verdades porque les sirven para representar el mundo que les rodea a partir de su capacidad de observación y de las experiencias vividas en su entorno. Una de las tareas del docente es moldear las ideas previas de los niños no cambiándolas por las ideas científicas, sino que el alumno sea capaz de reflexionar sobre ellas y plantearse preguntas de análisis que les ayuden a sustituirlas o simplemente corroborar las existentes complejizando los conceptos, entender que se dará "un proceso gradual de enriquecimiento y reestructuración de las estructuras conceptuales de los alumnos, de su manera de ver el mundo (Martín del Pozo, 2013).

\section{Procesos de experimentación en niños de edad preescolar}

El método científico es un conjunto de técnicas para investigar un fenómeno y adquirir nuevos conocimientos, así como para corregir e integrar conocimientos previos. Se basa en colectar evidencia observable, empírica y sujeta a los principios de razonamiento. (Contreras 2004).

Como su nombre indica representa la metodología que define y diferencia el conocimiento de la ciencia de otros tipos de conocimientos. Es un proceso en el cual se usan experimentos para contestar preguntas. El método científico, no está constituido por instrucciones mecánicas e infalibles, ni tampoco nos brinda demostraciones absolutas e invariables, sino que es dinámico, fundamentado en la propia práctica de su aplicación. El método científico es un proceso dinámico, que envuelve al observar todo el tiempo, buscar información continuamente $\mathrm{y}$ planificar experimentos para demostrar las hipótesis planteadas.

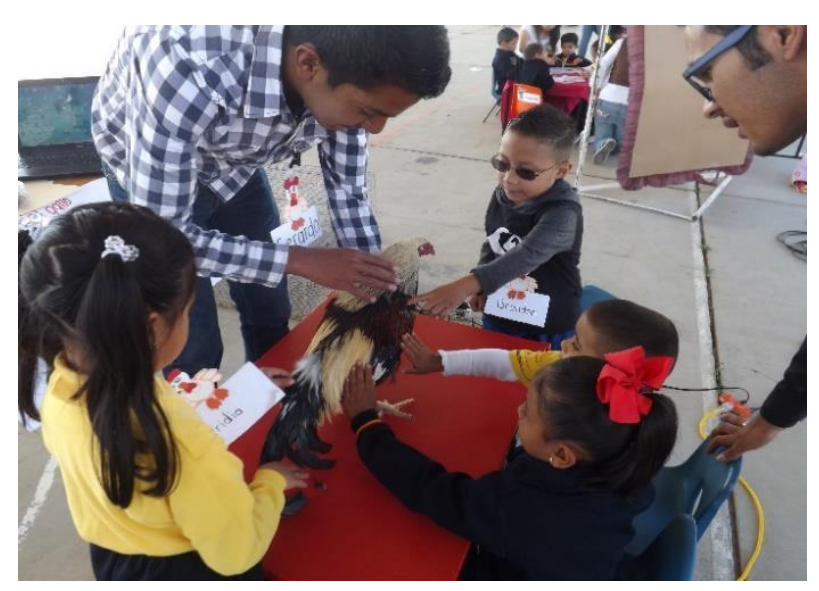

Figura 5 La observación y la experimentación ayudan a los niños a desarrollar su pensamiento científico

Este método de investigación, tiene una serie de pasos:

Observar e investigar.

Plantearse una pregunta o problema.

Establecer una posible respuesta a la pregunta. (Mejor conocida como hipótesis.)

BALDERAS-MIRELES, Ma. Dolores, ALMARAZ-OLGUÍN, Ma. Leticia, RAMÍREZ-VAQUERA, Irma y BALDERAS-MIRELES, Kathia María Antonieta. El aprendizaje científico en el niño de preescolar: una experiencia dinámica. Revista de Educación Básica. 2020 
Realizar la investigación necesaria (experimentar, recopilar datos, buscar información). Llegar a una conclusión, que apoye o refute la hipótesis.

Los niños en edad preescolar, los cuales aún no entran en la etapa de operaciones concretas planteadas por Piaget simplemente trabajan los pasos del método Científico de manera más simple, pero siguiendo el mismo orden:

- $\quad$ Observan lo que está sucediendo.

- Clasifican u organizar información.

- $\quad$ Predicen lo que sucederá.

- Comprueban predicciones bajo condiciones controladas para ver si son correctas.

\section{- Sacan conclusiones.}

Todos estos pasos son realizados por el niño de manera inconsciente, y se generan producto de la curiosidad constante que les acompaña. El último les permite construir sus explicaciones ante lo que ha ocurrido.

Por medio de los experimentos los niños logran por si solos, descubrir conceptos y verdades propias del medio circundante. Los beneficios de este tipo de actividades, es que buscan incentivar en el niño, el descubrir verdades absolutas, de forma divertida, incluso utilizando el juego como parte de este proceso y facilita su entendimiento.

Observar y experimentar son dos buenas formas para que los niños desarrollen su aprendizaje científico. Los contactos directos con las oportunidades de experimentación ayudan a los niños a razonar críticamente y sentirse más seguros de su propia habilidad para resolver problemas, dicha habilidad les ayudará a desarrollar conocimientos posteriores que posibilitan y potencian su aprendizaje.
Los niños de edad preescolar debido a sus características de pensamiento se interesan mucho en las cosas que pueden tocar, manipular y cambiar, al igual por las acciones que lleven implícito el juego, se convierte para ellos en una situación de aprendizaje motivadora; al igual las situaciones que les ayudan a descubrir qué sucede en cierto evento o fenómeno natural o social, se convierten en enigmas que los llevan a investigar, lo cual es el fundamento para el aprendizaje científico.

Es muy importante que se aliente a los niños a plantearse incógnitas, que se les hagan preguntas para hacerles compartir sus ideas $y$ que se preste atención al escuchar sus respuestas y brindar apoyo. No debemos olvidar que las experiencias que se les brindan a los niños les ayudan a formar sus ideas a encuadrarlas con o no con las últimas interpretaciones científicas, al igual a reforzar o modificar donde sea necesario hacerlo.

Al enseñar ciencias a los niños de preescolar no solo se están formando "futuros ciudadanos", es ampliar su horizonte de posibilidades cognitivas ellos como parte de una sociedad pueden actuar "de modo consciente y solidario respecto de temáticas vinculadas al bienestar de la sociedad de la que forman parte. (Daza R. y Quintanilla G. 2011).

Es importante incentivar a los niños a ver las cosas desde nuevas perspectivas. Las conversaciones que se entablen con ellos se pueden convertir en una forma importante de indagación y aprendizaje que más adelante se transformaran en un conocimiento científico permanente.

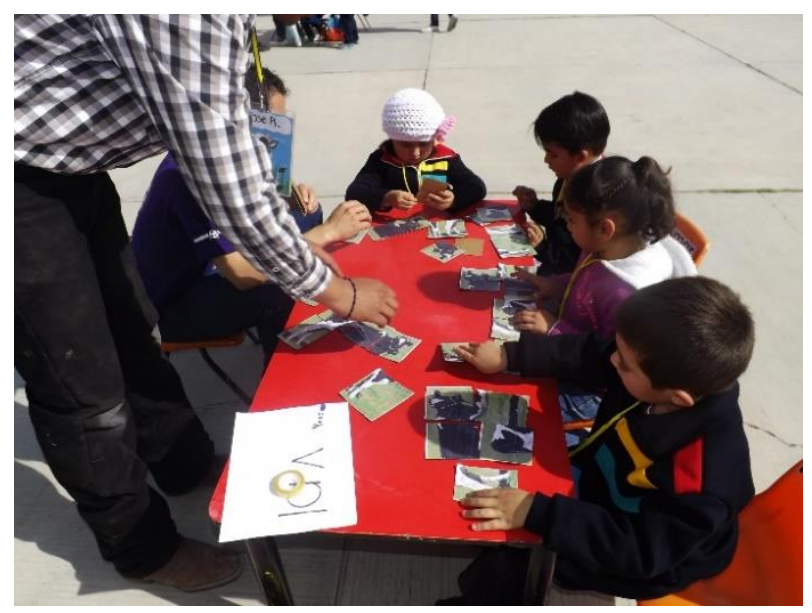

Figura 6 El maestro es quién guía las observaciones de los niños para lograr en ellos el aprendizaje que se ha propuesto 
Se debe impulsar al niño a desarrollar la capacidad de aprender a aprender y a satisfacer su curiosidad haciéndole saber que está bien si comete errores o reconocer que desconoce algo realizando una autoevaluación formativa $y$ retroalimentando lo ya existente. No es necesario decir: "No, esa no es la respuesta correcta," cuando él ofrece una explicación incorrecta se deben explotar sus propios saberes y hacerlo ver sus errores sin marcarlos para no influir en sus respuestas posteriores y castrar sus participaciones, se le debe ofrecer la información precisa y ayúdele a encontrar la respuesta acertada.

\section{Estrategia Predicción, Observación y Experimentación (POE)}

El desarrollo del aprendizaje científico en preescolar va encaminado a favorecer algunos apartados que comprenden el proceso del método científico como tal, no solo en este nivel educativo, sino que se prolongan a lo largo de la educación básica. Dentro de la educación preescolar se prioriza la observación, la experimentación y la construcción de inferencias por parte de los alumnos. Para ello es necesario ofrecer experiencias de contacto directo con los fenómenos que se vayan a abordar y en si con el medio ambiente natural en el cual se desarrollan los alumnos esto para abrir las posibilidades de desarrollar sus capacidades de reflexión y observación, al igual es importante brindarle la confianza necesaria para que externe sus pensamientos e ideas y así pueda predecir, formular inferencias y dar explicaciones de lo que ocurre en una determinada situación.

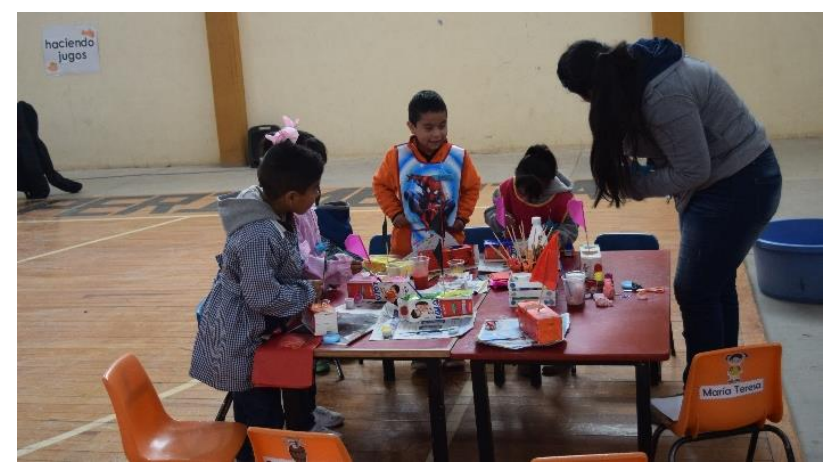

Figura 7 Las actividades que ofrecen los maestros de prescolar deben ayudar a desarrollar habilidades relacionadas con la ciencia como la formulación de hipótesis
En preescolar, el campo formativo se centra en el desarrollo del pensamiento reflexivo, y busca que los niños pongan en práctica la observación, formulación de preguntas, resolución de problemas y la elaboración de explicaciones, inferencias y argumentos sustentados en las experiencias directas; en la observación y el análisis de los fenómenos y procesos perceptibles que les ayudan a avanzar y construir nuevos aprendizajes sobre la base de los conocimientos que poseen y de la nueva información que incorporan. (SEP, Plan de estudios 2011, 2011).

Para tal efecto es necesario mencionar por separado la concepción de cada uno de los elementos que integran la estrategia POE para su mayor comprensión y utilización dentro de la educación preescolar; se sugiere la utilización de esta estrategia como parte del trabajo a realizar para mejorar la intervención docente.

Predicción: este rubro comprende cuando el alumno realiza una anticipación a lo que sucederá dentro de la situación y en él se formulan sus propias hipótesis a comprobar dentro de los pasos siguientes.

Observación: es una actividad innata del niño que se transforma en una herramienta de aprendizaje manipulable por el propio alumno y por el docente que guía el proceso.

Experimentación: se refiere a la fase dentro del método científico que consiste en manipular los materiales para obtener un resultado y poder así comprobar las hipótesis planteadas en la primera etapa de esta estrategia.

La enseñanza debe responde al deseo nato que los niños tienen por descubrir el mundo que les rodea propiciando, dentro o fuera del aula, experiencias significativas que provoquen placer por hacer y aprender ciencia (Perú Ministerio de educación, 2014).

Es importante proporcionarle al alumno las oportunidades para observar y experimentar con situaciones reales al alcance de los alumnos que los ayuden a enriquecer sus conocimientos. 
La experimentación puede considerarse como una herramienta enriquecedora en el campo Exploración y Comprensión del Mundo Natural pues brinda al alumno un sinfín de oportunidades para ampliar sus conocimientos y pasar de las concepciones erróneas o ideas previas a las concepciones verificadas por ellos mismos.

La observación como tal es una capacidad innata del ser humano más aun en los niños pequeños ya que se da de manera natural para satisfacer una curiosidad dirigida a un aspecto por conocer que sea de su interés, dentro de este aspecto es muy importante las estrategias de observación que utiliza el maestro como parte de su intervención docente no es sólo observar por observar.

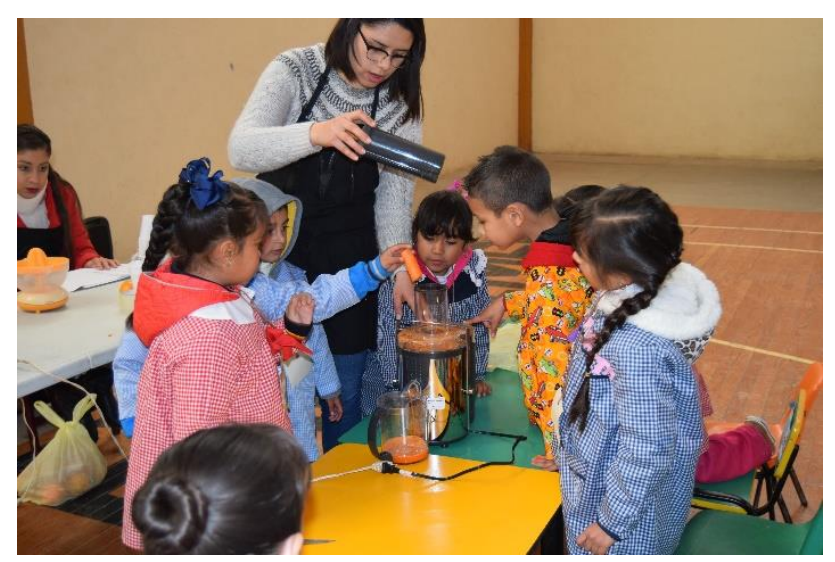

Figura 8 Los niños desarrollan la observación en las actividades que diseña la maestra de preescolar

Las niñas y los niños aprenden a observar cuando enfrentan situaciones que demandan atención, concentración e identificación de características de los elementos o fenómenos naturales. En la medida en que logran observar con atención, aprenden a reconocer información relevante de la que no lo es. (SEP, Programa de Educación Preescolar 2011 Guía para la educadora, 2011).

Entre las estrategias se puede utilizar una serie de preguntas que sirvan como guía para orientar a los alumnos encausándolos a un aspecto más específico; las consignas que se vayan a dar deben estar encausadas a la detección de los detalles, a la descripción a la comparación de los elementos de estudio en cuestión.
Esta intervención debe propiciar la construcción de inferencias y posibles explicaciones que más adelante ayudarán a los alumnos a formularse explicaciones propias de los fenómenos, a interiorizar la información, en un futuro a la asimilación de información y sobre todo la contrastación de las ideas y el remplazo de las ya existentes.

\section{Metodología}

La investigación se realizó bajo un enfoque cualitativo con alcance explicativo, se inicia con la aplicación de situaciones didácticas como parte de una evaluación diagnostica para de ahí partir con la aplicación y seguimiento de las actividades a desarrollar dentro de la propuesta.

Participó un grupo experimental el $1^{\circ} \mathrm{B}$ conformado por 25 alumnos, del Jardín de Niños "Ma. Enriqueta Camarillo de Pereyra" ubicado en Nieves, Gral. Francisco R. Murguía, Zacatecas.

Las actividades que se diseñaron y aplicaron como parte de las situaciones didácticas se encaminaron a favorecer el aprendizaje científico de los alumnos a partir del involucramiento de los padres de manera directa, mismas que llevaron a cabo con un acercamiento experimental y práctico por parte de ellos. En un segundo momento se diseñaron $\mathrm{y}$ aplicaron estrategias didácticas con actividades específicas encaminadas a favorecer el pensamiento científico con los alumnos del grupo.

Como instrumentos para recabar información durante las actividades, se utilizaron videos, fotografías y una guía de observación en la que los indicadores sobre el aprendizaje científico se organizaron en las siete habilidades que los niños de preescolar deben desarrollar, como técnica se empleó la observación.

También se aplicó un cuestionario tipo encuesta a los docentes de la zona escolar 06 con el propósito de saber los conocimientos que ellos poseen con respecto al pensamiento científico en el que se integraron 23 ítems y sobre los ambientes de aprendizaje apropiados para trabajar la ciencia con los niños de preescolar, integrado por 18 ítems. 
Se realizaron observaciones en los grupos de primer grado de tres jardines de niños pertenecientes a la zona escolar 06, durante éstas se utilizó la guía mencionada anteriormente, además se empleó la grabación en video y fotografías en apoyo al trabajo de investigación.

\section{Resultados}

Con respecto a las ideas previas que tienen los alumnos en relación al aprendizaje científico, es decir, al uso de la información que poseen los niños sobre determinado aspecto que se desea retomar dentro del salón de clase como antesala para la adquisición de nuevos conocimientos; se encontró (ver gráfico 1) que el nivel de exploración de las mismas es relativamente poco. Es importante conocer lo que los alumnos saben antes de iniciar con las actividades por ser punto primordial para el desarrollo de las acciones subsecuentes.

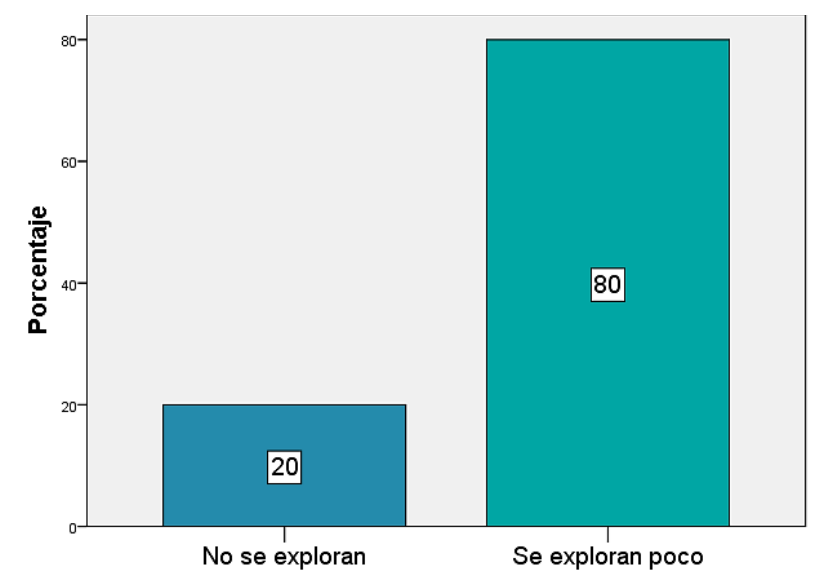

Gráfico 1 Ideas previas. Fuente propia a partir de resultados

Como parte de la planificación de clase y de la realización se debe tomar en cuenta el uso de materiales ya sean comerciales, tecnológicos o elaborados, los cuales deben reunir ciertas características acordes al nivel cognitivo de los alumnos con quiénes se vayan a utilizar (color, material usado para su elaboración, seguridad durante su manipulación, tipo y tamaño de letra, textura, adecuación a la edad) además deben encaminarse a favorecer el desarrollo de los aprendizajes esperados y el logro de los estándares curriculares.
El uso adecuado y pertinente de los materiales, así como su estructura de elaboración deben permitir el enlace entre los conocimientos previos y la construcción de los nuevos conocimientos. En el gráfico 2 se puede observar que el $6.66 \%$ de los docentes utilizan un material con características poco adecuadas al nivel preescolar, un 13.33 utilizan materiales no adecuado y el $80 \%$ del total de los encuestados no utilizan material por lo que queda descartado el nivel óptimo de utilización. La inexistencia de los materiales en las actividades áulicas en las que se pretende el aprendizaje científico queda con un nivel bajo desde la programación de situaciones didácticas.

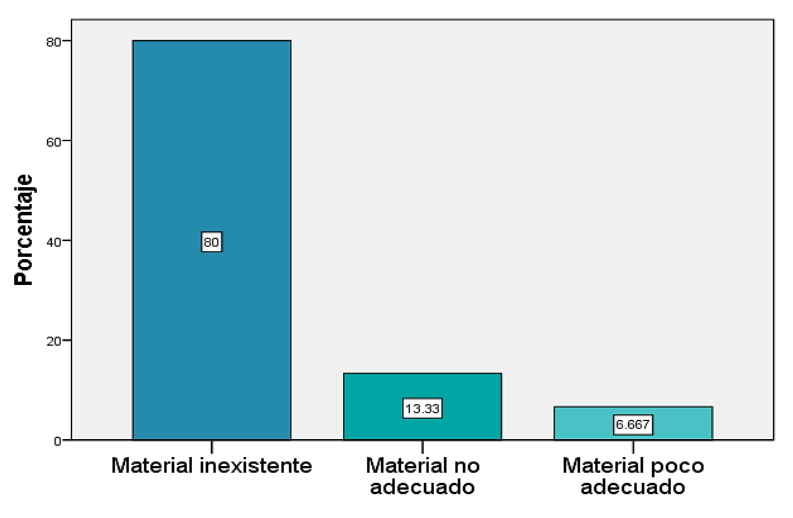

Gráfico 2 Características del material. Fuente propia a partir del análisis de resultados

Otro de los aspectos explorados fue el de la relación alumno-alumno, durante las experiencias de trabajo para el aprendizaje científico debe existir un clima de confianza y respeto que se refleje en el trabajo cotidiano, esto ayudará a que las actividades que se lleven a cabo sean exitosas en su totalidad, es en éstas donde los alumnos adquieren seguridad $\mathrm{y}$ confianza para externar sus conocimientos ante los demás, al igual que sus dudas sobre lo que se esté trabajando. Son parte de las habilidades de comunicación social que lo preparan para una vida adulta dentro de una sociedad integradora.

En el gráfico 3, se observa el sentir de los docentes con respecto a la relación alumnoalumno, un $93.33 \%$ de los docentes encuestados opina que es mala, reflejándose esto en la poca colaboración entre ellos y el trabajo en equipo que no se manifiesta como debiera ser para lograr un aprendizaje científico exitoso y eficaz. 


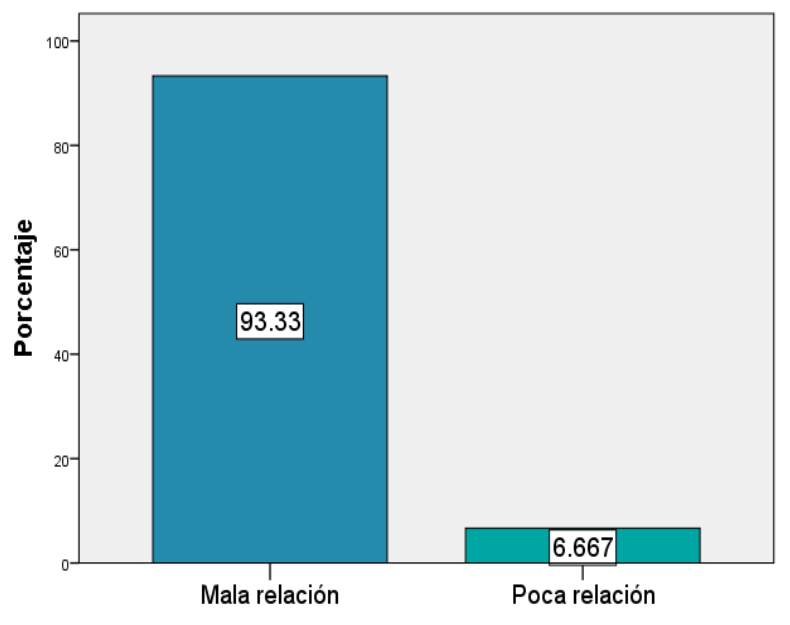

Gráfico 3 Relación alumno-alumno

Con respecto a las habilidades para el desarrollo del aprendizaje científico, se encontró que:

En las situaciones de observación los alumnos lo hacen con detenimiento, están atentos en el objeto de estudio, describen todo lo que observan, resaltando las características físicas de forma, color y tamaño.

En cuanto a la capacidad de asombro está más desarrollada en los alumnos de los jardines de niños ubicados en las comunidades que en los de la cabecera municipal, a estos últimos nada les parece novedoso, el docente tiene que apoyarlos con cuestionamientos o resaltando algún aspecto para que fijen su atención. Los alumnos de las comunidades se asombran y resaltan todos los aspectos de las situaciones didácticas que se trabajan, dejándolo notar por medio de los cuestionamientos que realizan.

$\mathrm{Si}$ a los alumnos se les permite tener contacto directo con los objetos de estudio, desde tocar, o poner en juego cualquiera de sus sentidos, lo levantan y manipulan con sus dedos para observarlo mejor, esto provoca que se mantenga el interés por un lapso mayor a diferencia de cuando no se les permite acercarse al objeto estudiado.

Las explicaciones que los propios alumnos dan son de manera empírica dejando en claro sus ideas, esto se manifiesta más en los alumnos de las comunidades que en los niños de los jardines urbanos, estos últimos se dejan guiar por lo que han observado en la tecnología mezclando sus ideas con las experiencias que traen desde sus hogares.
Los docentes encauzan esas explicaciones con intervenciones pequeñas relacionadas con el tema y guían a los alumnos a apoyarse en lo que observan de manera directa o en lo que ya conocen del tema o de la situación de aprendizaje.

Con respecto a las predicciones los alumnos realizan cuestionamientos sencillos al docente, basados en lo que conocen de manera empírica, dan respuestas breves anticipando lo que creen que sucederá en las actividades; dichas repuestas se basan más en conocimiento que les brinda el medio. Para algunos temas se dejó de tarea realizar investigaciones sobre aspectos y términos a usar dentro de las actividades. Esto influyó para que las respuestas de los alumnos se basaran en conocimientos científicos; aun así, al momento de realizar las actividades, las predicciones eran empíricas y hasta después modificaban sus respuestas.

En cuanto a las habilidades para la resolución de problemas, se encontró que los registros que realizan los alumnos son gráficos y presentan como características el garabato. Mezclando grafías, monigotes, trazos y colores. Para su interpretación el docente cuestiona de manera personal a los alumnos para lograr que externen lo que plasmaron en sus producciones.

Las clasificaciones que los alumnos realizan se basan en las características físicas observables de los objetos. No se presenta el concepto de reversibilidad ya que aún no se ha logrado desarrollar.

Los alumnos comprenden las características físicas de la materia que son observables dentro de algunos elementos o sustancias comunes para ellos ya que aquello que no observan directamente, difícilmente lo aceptan y comprenden. Para ello es necesario que hayan adquirido los conceptos de evocación de eventos y de temporalidad.

Las descripciones que los alumnos realizan de lo que ejecutan dentro de una situación de aprendizaje (visita, experimentación, observación, manipulación) son sencillas basadas en lo que más les llama la atención y complementando con lo que sus compañeros manifiestan en este aspecto. Los alumnos usan un tono de voz baja manifestando inseguridad al hacerlo. 
Siempre se guían por las preguntas que el docente realiza en relación con el trabajo. Las descripciones de lo que observan son cortas.

Las argumentaciones por parte de los alumnos se basan en lo que conocen, han escuchado o visto en algún medio de comunicación o dentro de su seno familiar. Para ellos la argumentación más acertada es la que involucra a sus familias especialmente a sus madres ya que ellas siempre tienen la razón y lo manifiestan diciendo "mi mamá me lo dijo" no aceptan poner estas respuestas en tela de juicio por parte del docente o de algún compañero del grupo. Los docentes guían las ideas de los alumnos por medio de preguntas clave que refuerzan las aseveraciones de los alumnos.

\section{Agradecimientos}

Agradecemos a las autoridades de los Jardines de Niños: "Ma. Enriqueta Camarillo de Pereyra", "José Vasconcelos", "Jacobo Luis Daguerre", así mismo a los docentes que participaron en la encuesta; todos de la zona escolar 06 de Francisco R, Murguía, por haber brindado las facilidades para este trabajo y a quienes fueron materia disponible, los niños de primer año grado.

\section{Conclusiones}

La mayoría de los docentes de educación preescolar, al ejecutar situaciones didácticas encaminadas a favorecer el aprendizaje científico en los alumnos, presentan deficiencias en la utilización del lenguaje apropiado usando un lenguaje coloquial sin fundamentos científicos.

Los docentes de educación preescolar abordan poco las situaciones didácticas que favorecen el desarrollo del aprendizaje científico en sus alumnos esto debido a que requieren una fundamentación científica pertinente sobre la información que deberán proporcionar.

En la educación preescolar el favorecimiento del aprendizaje científico es de gran importancia ya que este aspecto ayuda al desarrollo de competencias para la vida.
Con el desarrollo de situaciones de aprendizaje del campo de exploración y conocimiento del mundo los docentes favorecen el desarrollo del aprendizaje científico en los alumnos apoyándolos a ser analíticos, críticos, reflexivos, a que desarrollen su creatividad, experimenten, jueguen y manipulan. De igual forma aprenden a enfrentar y resolver problemas que se les presenten en la vida cotidiana, formulan supuestos y explicaciones argumentadas sobre el mundo que les rodea.

La utilización adecuada de los recursos y materiales didácticos dentro del campo de exploración y conocimiento del mundo permite motivar a los alumnos a conocer más sobre el mundo que les rodea y así contribuir al favorecimiento del aprendizaje científico.

Existe un tránsito del lenguaje empírico de los niños a un lenguaje científico una vez que se han aplicado las actividades planificadas para el aprendizaje del mismo

El uso técnico del lenguaje científico en los niños se fortalece, este se aprecia una vez que ponen de manifiesto las habilidades del desarrollo científico en las presentaciones.

Los niños fortalecen las habilidades para exponer ante el grupo de iguales o inclusive ante los padres de familia.

Son capaces de argumentar los resultados de las actividades que realizan dentro como fuera del aula.

\section{Referencias}

Ausbel,D. (1983). Teoría del aprendizaje significativo. Fásciculos de CEIF, 1-10.

B., B. (2005). Enseñar aentender lo que se lee. Buenos Aires, Argentina: Fondo de cultura economico de Argentina.

Bello, S. (2004). Ideas previas y cambio conceptual. Educación química, 2010 - 2017.

Daza Rosales S. y Quintanilla Gatica.2011. La enseñanza de las ciencias naturales en las primeras edades. Santiago de Chile. Litodigital.

Enciclopedia de la psicopedagogia . (2007). Barcelona, España: OCEANO. 
Manual de experimentos. (2007). Querétaro, Qro.: CONCYTEQ.

Martín del Pozo (coord.). (2013). Las ideas científicas de los alumnos y alumnas de Primaria: tareas, dibujos y textos. Madrid, España. GAMAR.

Meece, J. (2001) Desarrollo del niño y el adolescente. Compendio para educadores. Secretaría de Educación Básica y Normal.

Mendoza Reyes, M. d., y Silva Castillo, E. (2005). Modulo 3. En E. M. Sanchez, Curso de formacion y actualizacion profesional para el personal docente de educacion preescolar. Mexico: Imprentor S.A. DE C.V.

Ministerio de Educación. (2015). Rutas del aprendizaje Versión 2015. ¿Qué y cómo aprenden nuestros niños y niñas?. II Ciclo. Lima Perú

Pórlan Ariza, R., Rivero García, A., y Solís Ramírez, E. (2012). ¿Cómo representar el conocimiento currícular de los profesores de ciencias y su evolución? Enseñanza de las ciencias. investigación y experiencias didácticas

Quintanilla Gatica, M. 2017. Enseñanza de las ciencias e infancia. Problematicas y avance de teorías y campo desde Iberoamérica. Santiago de Chile. Editorial Bellaterra Ltda.

SEP. (2011). Plan de estudios 2011. Mexico, D.F. : CENTRO GRAFICO INDUSTRIAL S.A. DE C.V.

SEP. (2011). Programa de Educacion Preescolar 2011 Guía para la educadora. MEXICO: SEP.

Tonucci, F. (1995). Con ojos de maestro. Buenos Aires, Argentina: Troquel S.A.

Vigotsky, L. (1987). El niño y su saber y su saber hacer. México 